\title{
Classification of Handwritten Signatures Based on Name Legibility
}

\author{
Javier Galbally, Julian Fierrez and Javier Ortega-Garcia \\ Biometrics Research Lab./ATVS, EPS, Universidad Autonoma de Madrid, Campus de \\ Cantoblanco, C/ Francisco Tomas y Valiente 11, 28049 Madrid, Spain
}

\begin{abstract}
An automatic classification scheme of on-line handwritten signatures is presented. A Multilayer Perceptron (MLP) with a hidden layer is used as classifier, and two different signature classes are considered, namely: legible and non-legible name. Signatures are represented considering different feature subsets obtained from global information. Mahalanobis distance is used to rank the parameters and feature selection is then applied based on the top ranked features. Experimental results are given on the MCYT signature database comprising 330 signers. It is shown experimentally that automatic on-line signature classification based on the name legibility is feasible.
\end{abstract}

Keywords: Biometrics, classification, on-line signature, neural network, verification

\section{INTRODUCTION}

Thanks to its common use in many different applications, handwritten signature is one of the most widely accepted authentication methods. ${ }^{1} \quad$ It covers to some extent all of the main characteristics of a biometric trait (i.e., universality, distinctiveness, permanence and collectability ${ }^{2}$ ) so it is feasible to develop automatic recognition systems based on it. ${ }^{3,4}$

Two main approaches to the representation of signatures have been reported in literature $\left.{ }^{5}: i\right)$ off-line: in which only the final result (i.e., the image) of the signature is available, ${ }^{3}$ and $i i$ ) on-line: the signature is acquired with a special digitizing tablet which provides the temporal dynamics of the signature. ${ }^{4}$ In this paper we will focus on the latter approach, in which much richer information about the signature is available.

The present work is based on the so called occidental signatures. In opposition to other types of signatures consisting of independent symbols, such as the asian signatures, the occidental signatures consist of signs that could form concatenated text and some form of flourish. These can be classified according to different levels of complexity. In some countries the usual habit is to sign with readable written name, while in European countries signature must not necessarily have the readable written name of the signer and may only consist of an elaborated flourish. Examples of asian and occidental signatures can be found in the first International Signature Verification Competition. ${ }^{6}$ In Fig. 1 we depict different examples of signatures from the MCYT database, which is used in the experiments. In the top row of Fig. 1 we can see some signatures in which the name is non-readable. The bottom row of Fig. 1 shows some readable signatures.

Automatic signature verification systems have been shown to be sensitive to some extent to signature complexity. ${ }^{7}$ Recognition rates of some systems drop when coping with easy to forge signatures which are normally the readable ones. Therefore an automatic classification scheme to separate between legible and non-legible signatures would be desirable in order to improve the general performance of the verification system. In this case two different recognition strategies could be applied for legible and non-legible signatures thus maximizing the overall recognition rate.

Further author information: (Send correspondence to J.G.-H.)

J.G.: E-mail: javier.galbally@uam.es

J.F.: E-mail: julian.fierrez@uam.es

J.O.-G.: E-mail: javier.ortega@uam.es 

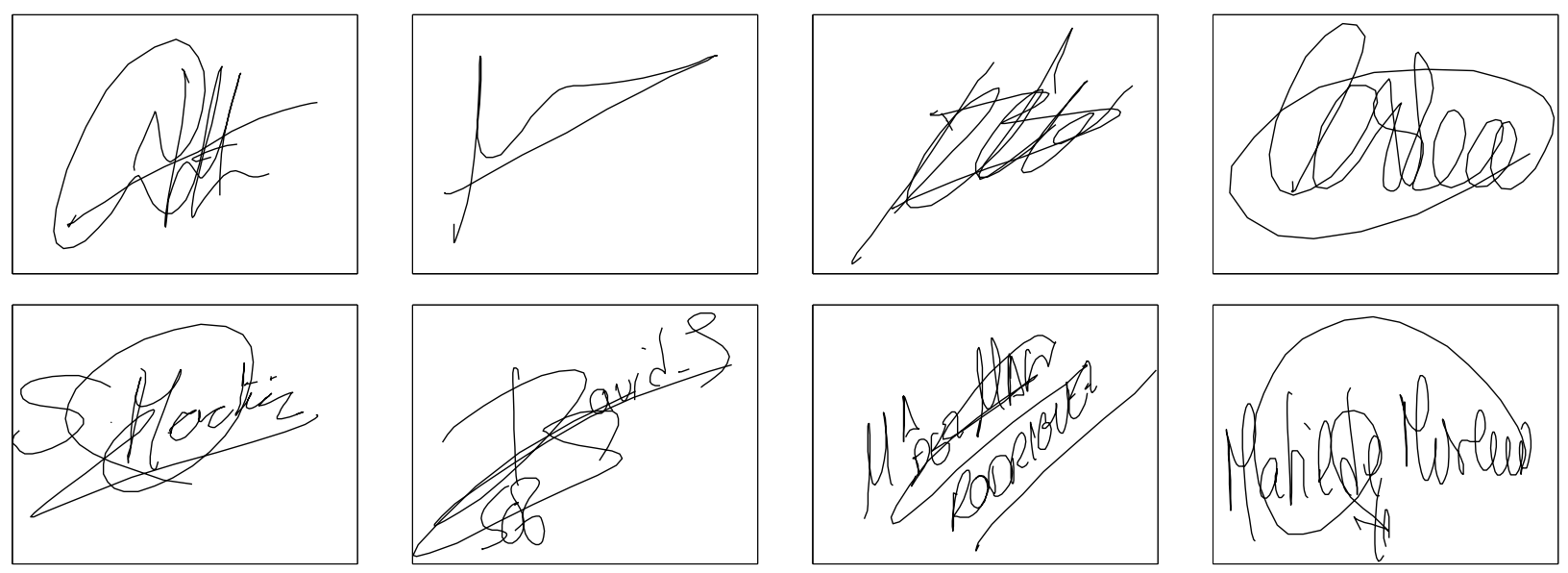

Figure 1. Signature examples of non-readable (top row) and readable signatures (bottom row).

From a privacy point of view, signatures in which the name of the signer is readable could involve a special treatment to protect the identity of the signer. From this perspective, an automatic legibility classification system would be relevant in order to adopt different measures for each signature depending on the specific legal guidelines for privacy protection of each country. ${ }^{8}$

This paper is structured as follows. Some related works are presented in Sect. 2. In Sect. 3 we detail the classification scheme followed, which is divided into feature extraction and selection, and neural network classifier. The experimental protocol, some results and further discussion are reported in Sect. 4. Conclusions are finally drawn in Sect. 5.

\section{RELATED WORKS}

Some similar efforts to the classification problem introduced in this paper have been made in previous works. $\mathrm{In}^{9}$ a classification scheme based on two consecutive classifiers is applied to handwritten text. In this work an initial pre-classification is made to discriminate between potentially problematic characters (such as ' $\mathrm{S}$ ' and ' 5 ') and then a specific adapted classifier is used for each one of these classes. In a similar way the present work proposes a pre-classification step which may be applied to signature verification in order to apply the most suitable recognition strategy to each signature class.

In ${ }^{10}$ a new authentication method based on written symbols is introduced and its discriminant capacity is compared to that of signatures. In one of the experiments reported a classifier is used for personal identification in a database in which symbols and signatures are mixed together. Although only one classifier is applied to the whole database, the process could be divided in two different steps: a first step in which symbols are separated from signatures (similar to the legibility classification problem introduced in the present work), and a second step in which each symbol/signature is recognized.

An automatic classification system for Tablet PC environments is presented in. ${ }^{11}$ In this work experiments using a HMM model are carried out in order to distinguish between graphics and handwritten text in on-line handwritten documents. This is the closest work to the proposed legibility classification scheme found in the literature.

\section{CLASSIFICATION SCHEME}

\subsection{Feature Extraction}

In the literature we can mainly find two different approaches to the problem of extracting relevant information from on-line signature data ${ }^{12}$ : 
Table 1. Set of global features considered in this work sorted by individual discriminative power. Some notation considerations are: $T$ denotes time interval, $t$ denotes time instant, $N$ denotes number of events, $v$ denotes velocity and $a$ denotes acceleration.

\begin{tabular}{|c|c|c|c|}
\hline Ranking & Feature Description & Ranking & Feature Description \\
\hline \hline $\mathbf{1}$ & signature total duration $T_{s}$ & $\mathbf{2}$ & $N($ pen-ups $)$ \\
\hline $\mathbf{3}$ & $N($ sign changes of $d x / d t$ and $d y / d t)$ & $\mathbf{4}$ & average jerk $\bar{\jmath}$ \\
\hline $\mathbf{5}$ & standard deviation of $a_{y}$ & $\mathbf{6}$ & standard deviation of $v_{y}$ \\
\hline $\mathbf{7}$ & (standard deviation of $y) / \Delta_{y}$ & $\mathbf{8}$ & $N($ local maxima in $x)$ \\
\hline $\mathbf{9}$ & standard deviation of $a_{x}$ & $\mathbf{1 0}$ & standard deviation of $v_{x}$ \\
\hline $\mathbf{1 1}$ & $j_{\text {rms }}$ & $\mathbf{1 2}$ & $N($ local maxima in $y)$ \\
\hline $\mathbf{1 3}$ & $t(2$ nd pen-down $) / T_{s}$ & $\mathbf{1 4}$ & $($ average velocity $\bar{v}) / v_{x, \max }$ \\
\hline $\mathbf{1 5}$ & $A_{\min }=\left(y_{\max }-y_{\min }\right)\left(x_{\max }-x_{\min }\right)$ & $\mathbf{1 6}$ & $\left(x_{\text {last pen-up }}-x_{\max }\right) / \Delta_{x}$ \\
\hline $\mathbf{1 7}$ & $\left(\Delta_{x}=\sum_{i=1}^{\text {pendowns }}\left(x_{\max \mid i}-x_{\min \mid i}\right)\right) \Delta_{y}$ & $\mathbf{1 8}$ & $\left(y_{\text {last pen-up }}-y_{\min }\right) / \Delta_{y}$ \\
\hline $\mathbf{1 9}$ & $\left(x_{1 \text { st pen-down }}-x_{\min }\right) / \Delta_{x}$ & $\mathbf{1 8}$ & $\left(T_{w} \bar{v}\right) /\left(y_{\max }-y_{\min }\right)$ \\
\hline
\end{tabular}

- Function-based approaches, in which time sequences describing local properties of the signature are used for recognition. ${ }^{13}$

- Feature-based approaches, in which a holistic vector representation consisting of global features is derived from the acquired signature trajectories. ${ }^{14}$

In the present work, in order to classify the on-line signatures based on name legibility, we focus on the latter approaches.

The device used in the signature acquisition process was a WACOM pen tablet which provided five different discrete-time dynamic sequences. In the experiments only features extracted from the first three sequences were used, the two involving angles were discarded as they have shown to be very unstable in some recognition systems. ${ }^{4}$ The five dynamic sequences are:

- Position in $x$-axis.

- Position in $y$-axis.

- Pressure $p$ applied by the pen.

- Azimuth angle of the pen with respect to the tablet.

- Altitude angle of the pen with respect to the tablet.

The complete set of global features used in the experiments is given in Table 1. This group of parameters is a subset of the features considered in. ${ }^{4}$ The work described in $^{4}$ was also carried out on the MCYT database, where users were asked to sign within a grid, so a controlled signature acquisition is assumed in order to reduce as much as possible the undesired effects produced by non-invariant translation/rotation features. In case of a non-controlled acquisition environment, translation/rotation registration should be performed before computing the feature set. The features in Table 1 are sorted according to the Mahalanobis distance criterion described in Sect. 3.2.

All the features were normalized to zero mean and unit variance before using them as the input to the classifier. 


\subsection{Feature Selection}

In order to check for possible improvements in the recognition rate depending on the number of features selected as input to the classifier, all the 20 parameters considered have been ranked according to scalar inter-user class separability as described in. ${ }^{4}$ Feature selection is then based on the top ranked features.

The ranking criterion is based on the maximization of expression

$$
S\left(F_{k}\right)=\sum_{i=1}^{330} \sum_{j=1}^{330}\left|d_{i, F_{k}}^{M}-d_{j, F_{k}}^{M}\right|,
$$

where $d_{i, F_{k}}^{M}$ is the Mahalanobis distance between the $F_{k}$-parameterized training signatures of client $i$ and the mean of the $F_{k}$-parameterized complete set of development signatures.

\subsection{Neural Network Classifier}

The proposed classifier is a Multilayer Perceptron (MLP) with one hidden layer of $M$ elements, and a backpropagation training scheme. The input layer is formed by $N$ elements (being $N$ the cardinality of the selected subset of features) with just two neurons in the output layer. The activation functions for each of the layers are:

- In the hidden layer a hiperbolic tangent: $f(u)=a \tanh (b \cdot u)$.

- In the output layer a sigmoid: $g(u)=1 /\left(1+e^{-u}\right)$.

In both cases a weighted linear combination is adopted for the net function of each neuron $i$ :

$$
u=\sum_{j=1}^{L} w_{j}^{i} y_{j}^{i}+\theta^{i}
$$

where $y_{j}^{i}$ is the $j$-th input of neuron $i, w_{j}^{i}$ the corresponding weight to that input, $L \in\{M, N\}$ is the number of elements of the previous layer and $\theta^{i}$ is the bias used to model the threshold.

The number of weights to be computed in the training of the MLP can be calculated as $N M+2 M$, being $M+2$ the number of bias elements.

In order to get linear performance of the classifier in the $[-1,1]$ range, the two parameters $a$ and $b$ of the hidden layer activation function were computed following the directives given in ${ }^{15}: a=1.716$ and $b=2 / 3$.

\section{EXPERIMENTS}

\subsection{Database}

MCYT bimodal database involves fingerprint and signature traits ${ }^{16}$ and it comprises 330 individuals acquired in the four institutions participating in the MCYT project. Approximately half of the donors were captured in the first institution and the other half in the remaining three. In the experiments carried out for the present paper only the signature subcorpus of the MCYT bimodal database is used.

Each of the 330 target users produced 25 genuine signatures, and 25 skilled forgeries. In this work we use all of the $330 \times 25=8250$ original signatures and no forgeries.

$\mathrm{In}^{16}$ an in depth description of the MCYT database with details of the software used and the acquisition process can be found.

Example data from the MCYT Signature database are shown in Fig. 2. 

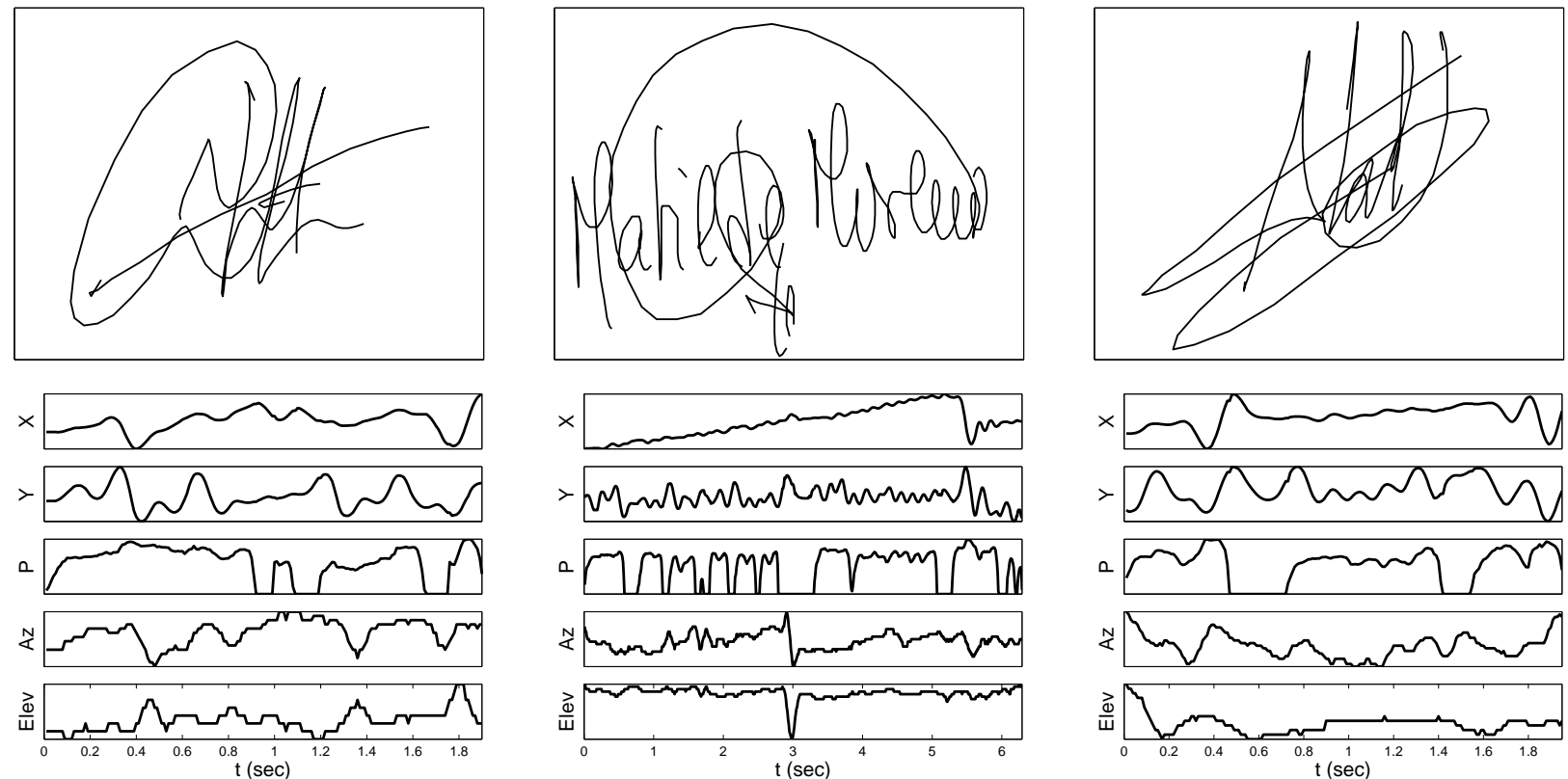

Figure 2. Signature examples from MCYT Signature database together with their dynamic sequences captured with a WACOM pen tablet.

\subsection{Experimental Protocol}

All the 8250 signatures considered in the experiments were labeled by hand as legible or non-legible. The network output $\left[O_{1} O_{2}\right]=\left[\begin{array}{ll}0 & 1\end{array}\right]$ was assigned to non-legible signatures (class $C_{0}$ ), and $\left[O_{1} O_{2}\right]=\left[\begin{array}{ll}1 & 0\end{array}\right]$ to legible signatures (class $C_{1}$ ). Both outputs of the Neural Network, $O_{1}$ and $O_{2}$, are real numbers in the $[0,1]$ range. The final decision rule is as follows:

- If $O_{1}<O_{2} \Rightarrow X$ is assigned to $C_{0}$

- If $O_{1}>O_{2} \Rightarrow X$ is assigned to $C_{1}$

where $X$ is the pattern (signature) to be classified.

Both classes are approximately a priori equally probable (162 signatures were manually assigned to class $C_{0}$ and 168 to class $C_{1}$ ). Note that the legibility of a signature is subjective and that two different persons might include the same signature in different classes. Thus, a perfect recognition rate can not be expected from any classifier.

The MLP classification performance measures have been computed using 2-fold cross-validation as follows:

1. A first recognition rate $T_{1}$ is computed using the first half of the patterns (i.e., patterns from 1 to 165 ) for the training of the Neural Network using the manually labeled ground truth, and the second half (that is patterns from 166 to 330 ) for testing.

2. A second recognition rate $T_{2}$ is computed the opposite way as $T_{1}$, i.e. the second half of the patterns is used for the training and the first half for the testing.

3. The final recognition rate of the classifier was obtained linearly combining the previous two,

$$
T=\frac{T_{1}+T_{2}}{2}
$$




\subsection{Results}

In Table 2 the recognition rates for increasing values of $N$ (number of features for each signature) and $M$ (number of elements in the hidden layer of the MLP) are given.

We first observe from Table 2 that up to $N=15$ the recognition rate increases with the number of features selected as input to the MLP. However, once this point is reached more input features worsens the classifier performance. This effect is depicted on the left plot of Fig. 3, where results for fixed $M=5$ are given. In this plot we can see a maximum in the performance of the classifier for $N=15$.

In Table 2 we can also observe an over-fitting effect, as the training set recognition rate increases with the number of elements of the hidden layer, while the recognition rate for the test set reaches a maximum for $M=5$ and then decreases. This effect is depicted in the right plot of Fig. 3 where the solid line shows the recognition rate on the test set (with a maximum in $M=5$ ) and the dashed line shows the recognition rate on the training set. These results were obtained with fixed $N=15$.

From the previous observations the best configuration of the MLP is $N=15$ and $M=5$. With this architecture a recognition rate of $78.66 \%$ is reached.

Further experiments for this same configuration were carried out changing the test and training sets selection scheme. Again a 2-fold cross-validation strategy was followed. In this case $T_{1}$ was computed using signatures from the odd users for training the neural network, and signatures from the even users as the test set. $T_{2}$ was computed exchanging both sets of data. This way we avoid the mismatch resulting from using all the signatures of the database acquired in one institution (the first 145 users, as described in Sect. 4.1) for the training and the signatures from other sites as testing data.

With this testing strategy the classifier performance was $\mathbf{8 4 . 5 6} \%$.

\subsection{Dicussion}

As remarked before the on-line signature legibility classification problem is subject to human opinion: even two different experts would not label all signatures in the same class. As an example signatures in Fig. 4, which were wrongly classified by the MLP, do not clearly belong to either of the classes. Signatures from the top row were manually labeled as non-legible, however some signs that could be interpreted as readable text can be observed. The classifier assigned them to class $C_{1}$ (legible). Although the name is not clearly legible, signatures from the bottom row were labeled as legible while the MLP interpreted them as non-legible.

\section{CONCLUSIONS}

An automatic on-line signature classification system was presented. Two different classes were considered, namely: legible and non-legible, and a Multilayer Perceptron (MLP) with a hidden layer was used as classifier. Signatures were parameterized considering different feature subsets obtained from global information and results were given on the whole MCYT signature database (330 signers).

It has been shown experimentally that automatic on-line signature legibility classification is feasible. In order to increase recognition rates the system is being improved by the incorporation of local information of the signatures. This type of system could be helpful as a pre-processing step in signature verification systems or in privacy-preserving related applications.

\section{ACKNOWLEDGMENTS}

J. G.-H. is supported by a FPU Fellowship from the Ministerio de Educacion y Ciencia (Spanish Ministry of Science) and J. F.-A. is supported by a FPI Fellowship from Comunidad de Madrid. This work was supported by the Spanish Ministry of Science and Technology under project TIC2003-08382-C05-01 and the European Network of Excellence Biosecure (IST-2002-507634). 
Table 2. Classification performance of the MLP for increasing values of $N$ (number of features) and $M$ (number of elements in the hidden layer). Recognition rates on the test set appear in bold and on the train set in plain text.

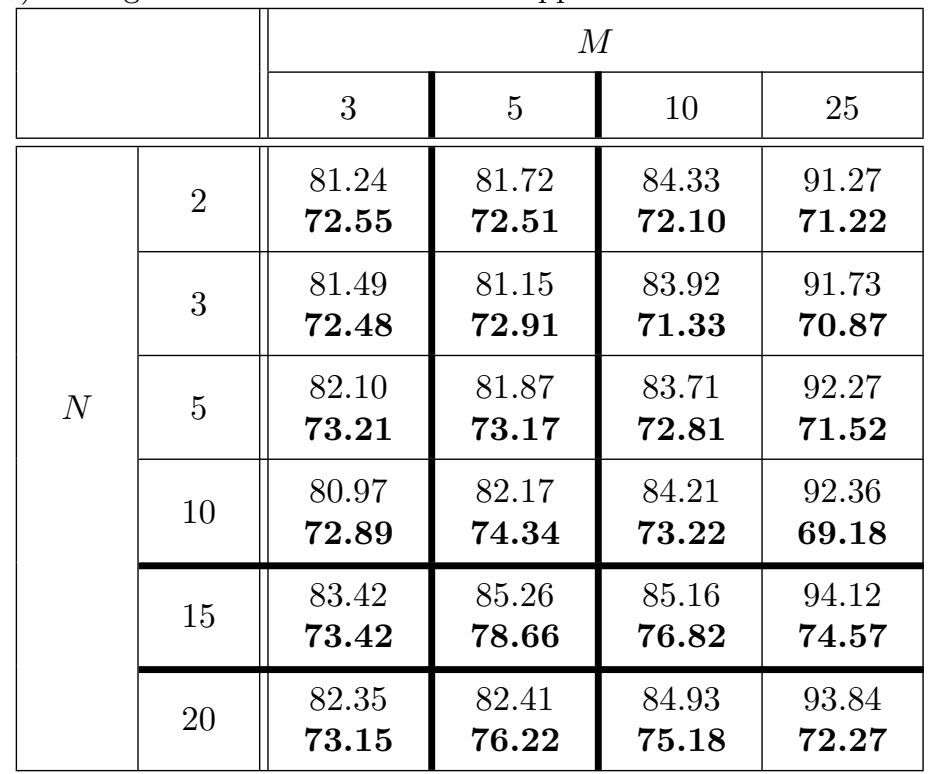
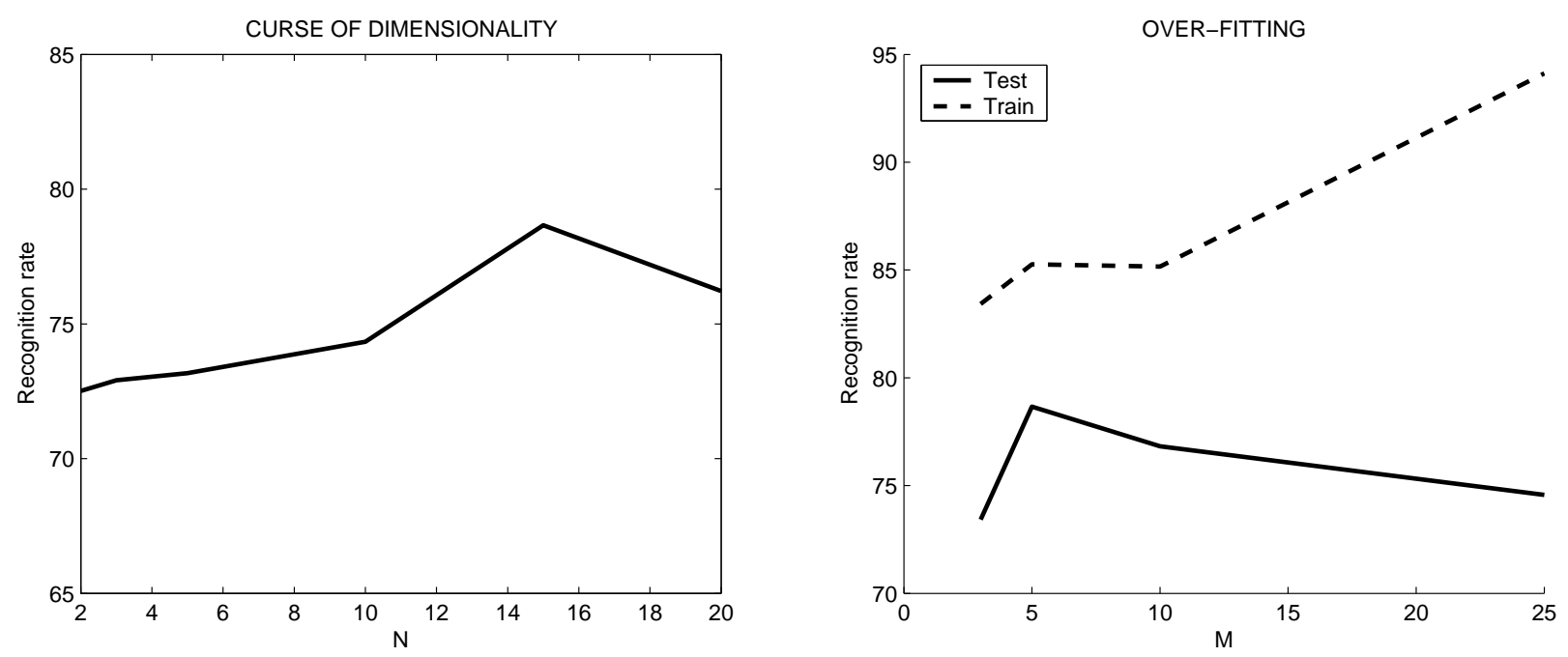

Figure 3. Graphics of the curse of dimensionality (left) and over-fitting (right) effects observed in the experiments. 

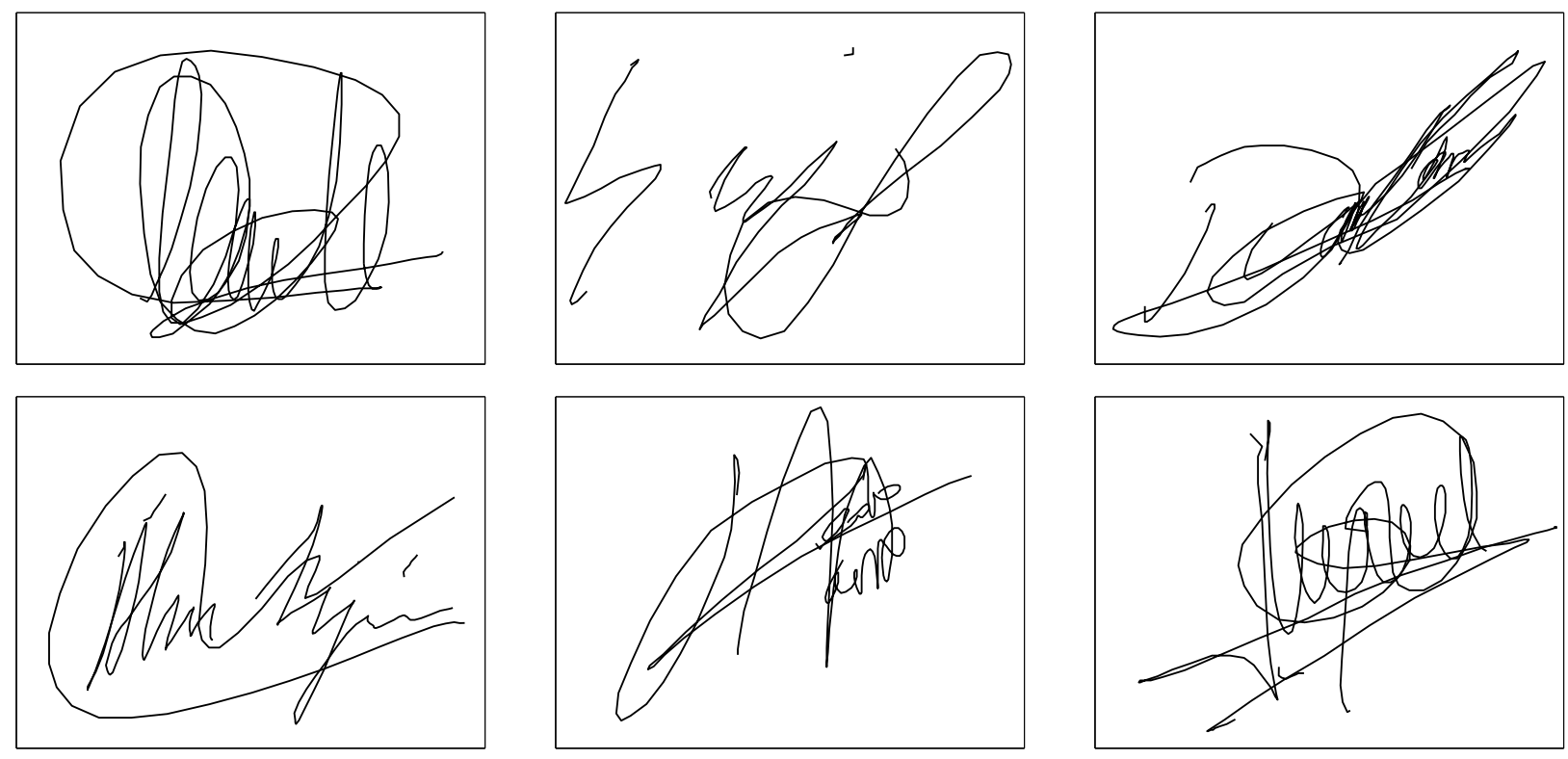

Figure 4. Signature examples wrongly classified using the best classifier configuration. In the top row signatures that were manually labeled in class $C_{0}$ (non-legible) and were assigned to class $C_{1}$ by the MLP are shown. The opposite error occurred on signatures shown in the bottom row.

\section{REFERENCES}

1. M. C. Fairhurst, "Signature verification revisited: promoting practical exploitation of biometric technology," Electronics and Communication Engineering Journal 9, pp. 273-280, December 1997.

2. A. K. Jain, A. Ross, and S. Prabhakar, "An introduction to biometric recognition," IEEE Trans. on Circuits and Systems for Video Technology 14, pp. 4-20, January 2004.

3. J. Fierrez-Aguilar, N. Alonso-Hermira, G. Moreno-Marquez, and J. Ortega-Garcia, "An off-line signature verification system based on fusion of local and global information," in BIOAW, LNCS-3087, pp. 295-306, Springer, 2004.

4. J. Fierrez-Aguilar, L. Nanni, J. Lopez-Penalba, J. Ortega-Garcia, and D. Maltoni, "An on-line signature verification system based on fusion of local and global information," in AVBPA, LNCS-3546, pp. 523-532, Springer, 2005.

5. R. Plamondon and S. N. Srihari, "On-line and off-line handwriting recognition: a comprehensive survey," IEEE Trans. on Pattern Analysis and Machine Intelligence 22, pp. 63-84, January 2000.

6. D.-Y. Yeung, H. Chang, et al., "SVC2004: First International Signature Verification Competition," in ICBA, LNCS-3072, pp. 16-22, Springer, 2004.

7. J. Fierrez-Aguilar, J. Ortega-Garcia, and J. Gonzalez-Rodriguez, "Target dependent score normalization techniques and their application to signature verification," IEEE Trans. on Systems, Man, and Cybernetics 35, pp. 418-425, 2005.

8. M. Rejman-Greene, Biometric systems: technology, design and performance evalutation, ch. Privacy issues in the application of biometrics: a European perspective, pp. 335-356. Springer, 2005.

9. M. Fairhurst and A. F. Rahman, "Generalised approach to the recognition of structurally similar handwritten characters using multiple expert clasifiers," IEE Proc. Vis. Image Signal Process. 144, pp. 15-22, February 1997.

10. M. G. Lizarraga and L. L. Ling, "Biometric personal authentication based on handwritten signals," in ICBA, LNCS-3072, pp. 533-539, Springer, 2004.

11. C. M. Bishop, M. Svensen, and G. E. Hilton, "Distinguishing text form graphics in on-line handwritten ink," in IWFHR-9, 2004. 
12. R. Plamondon and G. Lorette, "Automatic signature verification and writer identification: The state of the art," Pattern Recognition 22(2), pp. 107-131, 1989.

13. J. Ortega-Garcia, J. Fierrez-Aguilar, J. Martin-Rello, and J. Gonzalez-Rodriguez, "Complete signal modeling and score normalization for function-based dynamic signature verification," in Proc. AVBPA, LNCS2688, pp. 658-667, 2003.

14. L. L. Lee, T. Berger, and E. Aviczer, "Reliable on-line human signature verification systems," Pattern Analysis and Machine Intelligence 18, pp. 643-647, 1996.

15. S. Haykin, Neural networks: a comprehensive foundation, ch. Multilayer Perceptrons, pp. 156-255. Prentice Hall, 1999.

16. J. Ortega-Garcia, J. Fierrez-Aguilar, et al., "MCYT baseline corpus: a bimodal biometric database," IEE Proc. Vis. Image Signal Process. 150, pp. 395-401, December 2003. 